\title{
Introduction of Posterior Axilla Sling Traction in Simulated Shoulder Dystocia
}

\author{
Julie R. Whittington, MD, MC, USN ${ }^{1,2}$ Aaron T. Poole, MD, MC, USN ${ }^{1}$ \\ ${ }^{1}$ Division of MFM, Department of OB/GYN, Naval Medical Center \\ Portsmouth, Portsmouth, Virginia \\ 2 Division of MFM, Department of OB/GYN, University of Arkansas for \\ Medical Sciences, Little Rock, Arkansas

\begin{abstract}
Address for correspondence Julie Whittington, MD, MFM Fellow, University of Arkansas for Medical Sciences, 501 West. Markham Street. Slot 518, Little Rock, AR 72205 (e-mail: Julie.whittington09@gmail.com).
\end{abstract}

Am J Perinatol Rep 2018;8:e247-e250.

\begin{abstract}
Keywords

- simulation

- shoulder dystocia

- posterior axilla sling traction

- obstetric emergency

Objective Aim of this study was to introduce posterior axilla sling traction (PAST) in delivering providers and nursing staff as an adjunct to the management of shoulder dystocia and evaluate comfort in performing the maneuver.

Methods A presimulation questionnaire had given to all participants. A brief training on how to perform PAST was also given. A simulated shoulder dystocia was run where usual maneuvers failed. Participants used PAST for delivery of posterior shoulder, delivery of posterior arm, and to assist with rotation. Participants were then given a post-simulation questionnaire. A Chi-squared test was used to evaluate comfort with performing the procedure pre and post-simulation.

Results Data were collected from 43 participants at pre and post-simulation. Designations (attending, resident, midwife, registered nurse) and responses were recorded to the questionnaires. There was a statistically significant increase in the number of providers and nurses who would feel comfortable using PAST for shoulder dystocia management and for rotational maneuvers. Ninety-three percent of participants would consider using PAST in future shoulder dystocia when usual maneuvers failed.

Conclusion PAST is an adjunct to management of shoulder dystocia that has not previously been taught in our facility. The majority of participants in our simulation felt comfortable with using PAST.
\end{abstract}

Obstetricians are taught from the beginning of their training to prepare for the worst. Obstetric emergencies can be catastrophic for the patient, the infant, and the provider when poor outcomes occur. Shoulder dystocia is defined as failure to deliver the fetal shoulders with gentle downward traction, requirement of additional maneuvers to affect delivery, or a head to body interval of greater than 1 minute. ${ }^{1,2}$ Shoulder dystocia affects 0.6 to $1.4 \%$ of all vaginal deliveries. ${ }^{1}$ The rate of injury in shoulder dystocia has been reported at $24.9 \%$ including brachial plexus injuries, clavicular or humeral fractures, and fetal death. ${ }^{3}$ The rate of brachial plexus injury alone has been reported as ranging from 4 to $40 \%{ }^{4}$ Shoulder dystocia can be a daunting obstetric emergency, especially when traditional maneuvers have failed.

received

April 19, 2018

accepted after revision

September 12, 2018
DOI https://doi.org/

10.1055/s-0038-1675350. ISSN 2157-6998.
In the 2006, the Menticoglou's maneuver was introduced which uses the delivering provider's fingers in the posterior axilla for delivery of the posterior shoulder. ${ }^{5}$ This maneuver can be helpful when others have failed; however, space can be limited and the insertion of two fingers into the axilla would theoretically increase the bisacromial diameter. In the 2009, Hofmeyr and Cluver introduced using a suction catheter looped through the posterior axilla. Initially, it was described in two cases of shoulder dystocia following delivery of previously demised infants. ${ }^{6}$ Later that year, the authors described the use of posterior axilla sling traction (PAST) in a case series of three shoulder dystocias occurring with live births. ${ }^{7}$ Most recently, the authors described a series of 19 cases which utilized PAST; delivery was successful in 18 of the
Copyright $@ 2018$ by Thieme Medical Publishers, Inc., 333 Seventh Avenue, New York, NY 10001, USA. Tel: +1(212) 584-4662.
License terms

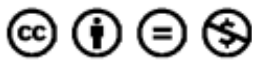


19 cases. In the other case, the use of PAST was thought to be partially successful. Rotation with the sling was also introduced as an additional maneuver when delivery of the posterior shoulder or arm with the sling was unsuccessful. ${ }^{8}$

The American College of Obstetricians and Gynecologists specifically recommends simulation for preparation for shoulder dystocia. Shoulder dystocia simulation has been shown to increase technical skills and communication skills for residents. ${ }^{9}$ Simulation of shoulder dystocia has also been shown in multiple studies to improve safety and reduce injuries including brachial plexus injuries. ${ }^{10}$

Posterior axilla sling traction has not previously been taught or utilized at Naval Medical Center Portsmouth. Our objective was to introduce posterior axilla sling traction to attending providers, residents, midwives, and nursing staff as an adjunct to the management of shoulder dystocia and evaluate comfort in performing the maneuver or performance of the maneuver by the clinician. We also wanted to evaluate any barriers to usage of this maneuver within our facility. Siassakos et al describe six elements of effective training for emergencies in obstetrics, one of which is multiprofessional teams with clinical and teamwork training. ${ }^{11}$ We felt it was important to include nursing staff in the introduction of PAST in order for them to feel comfortable with providers performing this procedure and also to voice ideas which may help in emergent situations.

\section{Materials and Methods}

A presimulation questionnaire was given to all participants. This questionnaire included their classification as an attending, resident, midwife, or nurse, along with an assessment of their prior usage or familiarity with PAST. We also assessed comfort and rotation with PAST.

We then gave a brief training on how to perform PAST. Initially, the technique was described to participants including delivery of posterior shoulder, facilitation of delivery of the posterior arm, and finally using the sling for rotation. It was then demonstrated to participants using a bony pelvis, a plastic baby, and a 14-French suction catheter.

A simulated shoulder dystocia was then initiated. The simulated patient (the Noelle's model) was a 35 year-old, gravida 2 para 1 , at 39 weeks of gestation who presented in active labor and was now pushing with her nurse. She had an epidural in place and her labor was not augmented. A provider was called for delivery. The infant's head delivered easily but the shoulders were unable to be delivered with gentle downward traction. The providers went through all the usual maneuvers which were not successful. Participants then used posterior axilla sling traction for delivery of posterior shoulder, delivery of posterior arm, and to assist with rotation. If needed, assistance was given as participants attempted these maneuvers. All participants were able to perform the maneuvers by the conclusion of the simulation. A debrief was then performed and the teams were able to ask questions of each other and of the instructors.

Participants were then given a post simulation questionnaire. Again, their classification was noted. Comfort with PAST
Table 1 Participants

\begin{tabular}{|l|l|}
\hline Staff provider & $10(23 \%)$ \\
\hline Resident & $11(26 \%)$ \\
\hline Midwife & $4(9 \%)$ \\
\hline Delivering providers & $25(58 \%)$ \\
\hline Nursing & $18(42 \%)$ \\
\hline
\end{tabular}

and rotation with the sling were assessed using the same scale as the preassessment questionnaire. We also asked participants if they would consider using or suggesting a provider use PAST in future shoulder dystocia situations when other methods fail. A Chi-squared test was used to evaluate comfort with performing the procedure pre and post-simulation.

This study was deemed nonhuman subjects research by the Naval Medical Center Portsmouth Institutional Review Board.

\section{Results}

Data were collected from 43 participants' pre and post-simulation. We recorded their designation (attending, resident, midwife, registered nurse) and their responses to the questionnaires. Fifty-eight percent of participants were delivering providers and $42 \%$ of participants were labor and delivery nurses (-Table $\mathbf{1}$ ).

Prior to simulation, $28 \%$ of all participants were familiar (answered disagree or strongly disagree on questionnaire) with PAST as an alternative maneuver for shoulder dystocia. Following the simulation, 91\% of participants would consider using PAST in future shoulder dystocia emergencies (agree or strongly agree; - Fig. 1). There was a statistically significant increase in the number of providers and nurses who would feel comfortable using PAST for shoulder dystocia with a $p<0.001$ (-Fig. 2). There was also a statistically significant increase in the participants who would feel comfortable using PAST for rotational maneuvers with a $p<0.001$ (-Fig. 3).

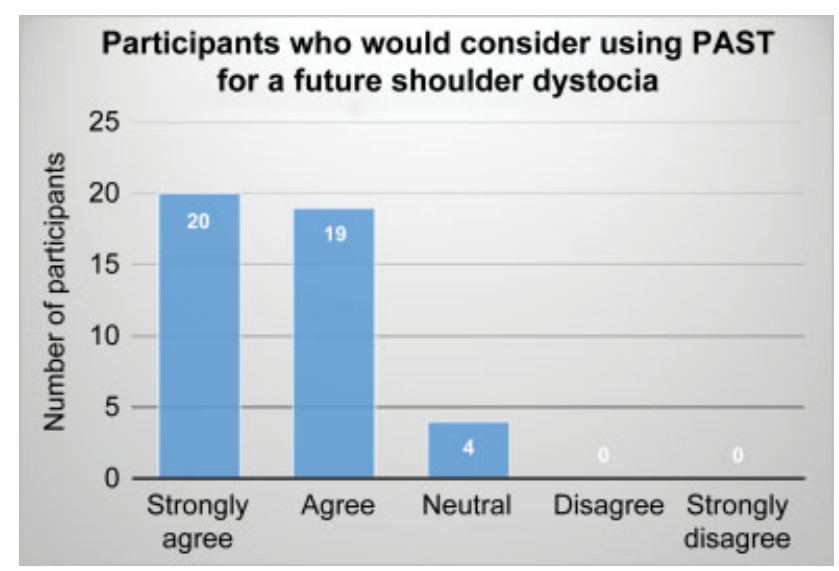

Fig. $139 / 43$ participants would consider using PAST in a future shoulder dystocia. Only 3/43 participants were neutral. There were zero respondents that disagreed or strongly disagreed that they would consider using PAST in a future shoulder dystocia. PAST, posterior axilla sling traction. 


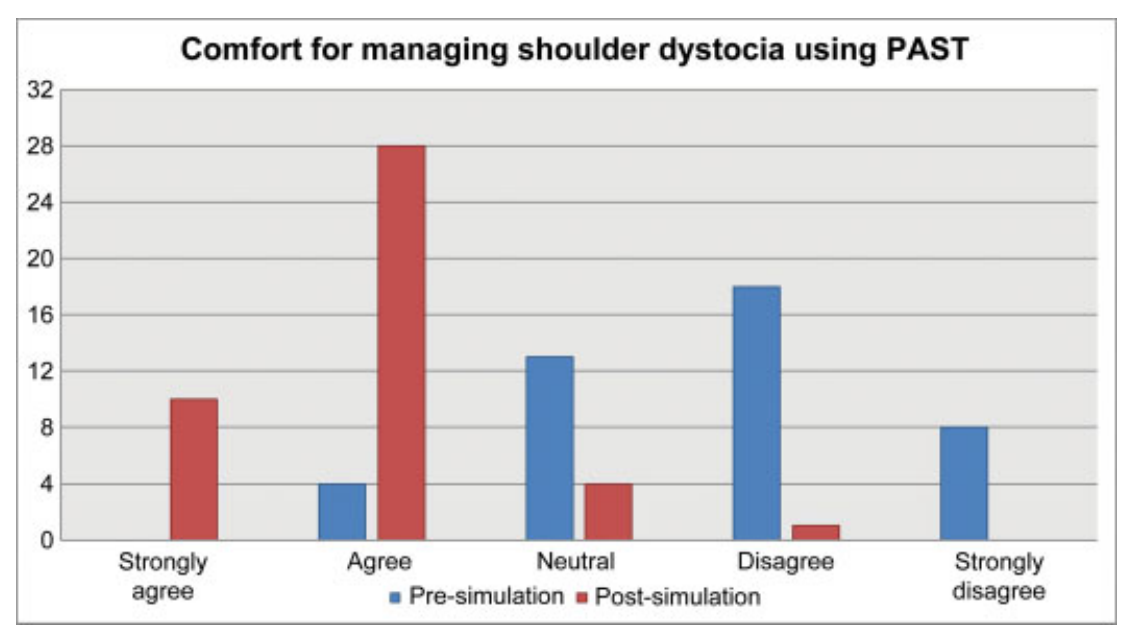

Fig. 2 Presimulation, 9\% of participants would be comfortable using PAST in clinical scenarios. After the simulation, 88\% felt comfortable using PAST for managing shoulder dystocia. $p<0.001$ for agree or strongly agree. PAST, posterior axilla sling traction.

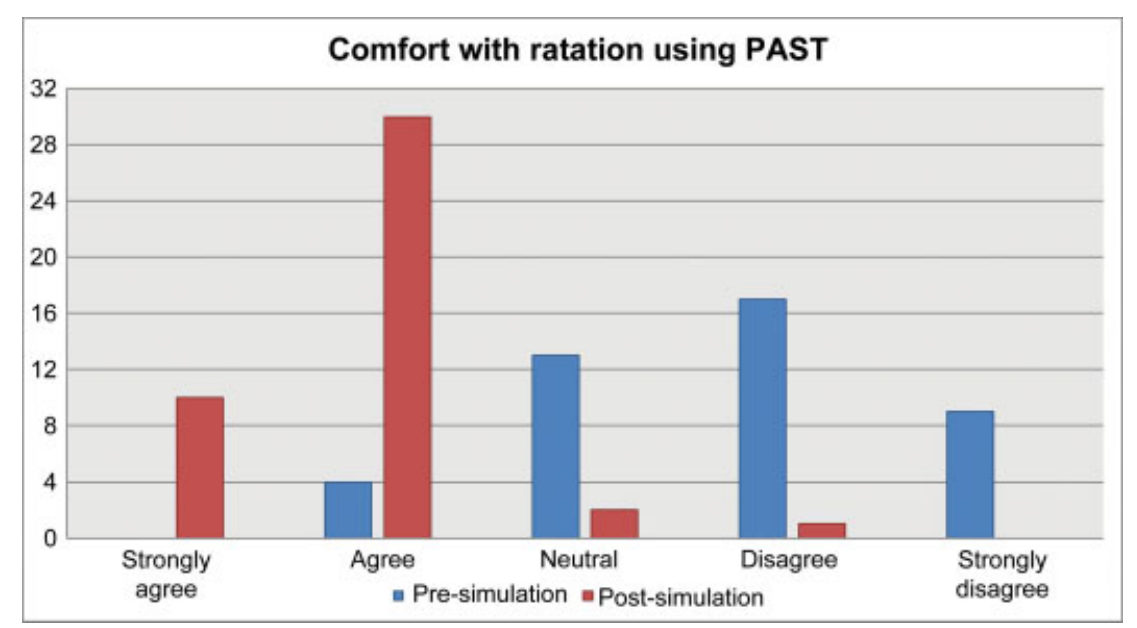

Fig. 3 Presimulation, 9\% of participants would be comfortable using PAST for rotational maneuvers. Post simulation, $93 \%$ of participants felt comfortable using PAST for rotational maneuvers. $p<0.001$ for agree or strongly agree. PAST, posterior axilla sling traction.

After simulation training, $70 \%$ of participants answered all goals of PAST correctly including delivery of posterior shoulder, delivery of posterior arm, and rotation. The most frequent barriers identified for implementation of PAST as an adjunct for management of shoulder dystocia at our facility were familiarity ( $16 \%$ of responses), availability of equipment ( $16 \%$ of responses), or both ( $55 \%$ of participants).

\section{Discussion}

Shoulder dystocia is an obstetric emergency that requires preparation and training for proper management by delivering providers. Not only does the baby need to be delivered expeditiously, care must be taken to minimize risk of injury to the mother and the infant. Simulation and practicing protocols can promote the team based approach to shoulder dystocia. ${ }^{12}$ When usual maneuvers have failed (including hyperflexion of the maternal legs, suprapubic pressure, internal rotational maneuvers, delivery of the posterior arm, and all-fours positioning), delivering providers need additional tools at their disposal. During our simulation, we did not have providers perform PAST until all other maneuvers were exhausted. PAST offers another method for delivery while further preparations are being made for an operating room.

PAST does require that materials needed for the maneuver (a suction catheter and a clamp) be readily available. ${ }^{13}$ To remedy this in our institution, suction catheters were placed in the top drawer of all of our delivery carts. A standard clamp is available on a delivery tray that works well. The clamp is placed across the two ends of the catheter and is used to secure the catheter; it also provides a handle for application of traction.

Participants in our briefing and simulation had a significant increase in their comfort with both use of PAST and use of the sling for rotation. Materials required for this simulation were minimal and PAST was an easily taught and assimilated skill.

Although safety of PAST is not well studied, in the case series of 19 patients by Hofmeyr and Cluver, the injuries to the posterior arm were isolated to five humeral fractures. There were also brachial plexus injuries to the anterior shoulder but given that is not where manipulation occurred, it was likely not due to the PAST maneuver. ${ }^{8}$ Further research is needed on the safety of PAST, therefore we agree with the 
authors that it should not be attempted until usual maneuvers have failed. $^{7}$

We involved our labor and delivery nurses in this exercise for multiple reasons, including promotion of a team-based approach to shoulder dystocia. When a new technique is introduced, it is helpful if members of the healthcare team are familiar with it, can help to locate materials needed if not readily available, and can suggest the use of the maneuver if the delivering provider did not consider it.

\section{Conclusion}

PAST is a useful adjunct to the usual maneuvers for shoulder dystocia as it can be used successfully when other maneuvers have failed. We do not recommend PAST as a first line maneuver and do not recommend using it until usual maneuvers have not relieved the shoulder dystocia. PAST was easily taught in our institution and the vast majority of participants would consider usage of PAST in future shoulder dystocia. By having materials readily available, we have removed one of the barriers to using PAST in a clinical situation. We advocate for more research on PAST. As more delivering providers become familiar with this technique, it will foreseeably be used more in the future and more data will be available on its safety and efficacy.

\section{Note}

The authors are military service members (or employee of the U.S. Government). This work was prepared as part of their official duties. Title 17, USC, $\S 105$ provides that 'Copyright protection under this title is not available for any work of the U.S. Government.' Title 17, USC, §101 defines a U.S. Government work as a work prepared by a military service member or employee of the U.S. Government as part of that person's official duties.

The views expressed in this article are those of the authors and do not necessarily reflect the official policy or position of the Department of the Navy, Department of Defense, or the United States Government.

Conflict of Interest

The authors report no conflict of interest.

\section{Paper Presentation}

Poster Presentation at the Society for Maternal Fetal Medicine Annual Meeting, Dallas, TX 2/3/2018

\section{Acknowledgments}

We would like to thank Ms. Donna Eastham, B.A., C.R.S. for her assistance with manuscript editing and preparation.

\section{References}

1 American College of Obstetricians and Gynecologists. Practice bulletin number 178: shoulder dystocia. ACOG 2017;129(05): e123-e133

2 Beall MH, Spong C, McKay J, Ross MG. Objective definition of shoulder dystocia: a prospective evaluation. Am J Obstet Gynecol 1998;179(04):934-937

3 Gherman RB, Ouzounian JG, Goodwin TM. Obstetric maneuvers for shoulder dystocia and associated fetal morbidity. Am J Obstet Gynecol 1998;178(06):1126-1130

4 Dajani NK, Magann EF. Complications of shoulder dystocia. Semin Perinatol 2014;38(04):201-204

5 Menticoglou SM. A modified technique to deliver the posterior arm in severe shoulder dystocia. Obstet Gynecol 2006;108 (3, Pt 2):755-757

6 Cluver CA, Hofmeyr GJ. Posterior axilla sling traction: a technique for intractable shoulder dystocia. Obstet Gynecol 2009;113 (2, Pt 2):486-488

7 Hofmeyr GJ, Cluver CA. Posterior axilla sling traction for intractable shoulder dystocia. BJOG 2009;116(13):1818-1820

8 Cluver CA, Hofmeyr GJ. Posterior axilla sling traction for shoulder dystocia: case review and a new method of shoulder rotation with the sling. Am J Obstet Gynecol 2015;212(06):784.e1-784.e7

9 Mannella P, Palla G, Cuttano A, Boldrini A, Simoncini T. Effect of high-fidelity shoulder dystocia simulation on emergency obstetric skills and crew resource management skills among residents. Int J Gynaecol Obstet 2016;135(03):338-342

10 Shaddeau AK, Deering S. Simulation and shoulder dystocia. Clin Obstet Gynecol 2016;59(04):853-858

11 Siassakos D, Crofts JF, Winter C, Weiner CP, Draycott TJ. The active components of effective training in obstetric emergencies. BJOG 2009;116(08):1028-1032

12 Moni S, Lee C, Goffman D. Shoulder dystocia: quality, safety, and risk management considerations. Clin Obstet Gynecol 2016;59 (04):841-852

13 Gilstrop M, Hoffman MK. An update on the acute management of shoulder dystocia. Clin Obstet Gynecol 2016;59(04):813-819 